\title{
Safinamide - A Unique Treatment Targeting Both Dopaminergic and Non-Dopaminergic Systems
}

\author{
Jaime Kulisevsky ${ }^{1,2,3}$ \\ 1. Movement Disorders Unit, Neurology, Hospital Santa Creu, Barcelona, Spain; 2. Estudis de Ciències de la Salut, Universitat Oberta de \\ Catalunya (UOC), Barcelona, Spain; 3. CIBERNED, Instituto de Salud Carlos III, Madrid, Spain
}

DOI: https://doi.org/10.17925/ENR.2016.11.02.101

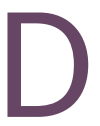
opaminergic replacement therapies are prescribed widely to improve motor problems in Parkinson's disease (PD). However, as the disease progresses, the response to levodopa (I-dopa) doses becomes shorter and patients experience symptom recurrence at the end of the dose effect. These so-called OFF periods may become refractory to treatment, and may become associated with disabling motor fluctuations or dyskinesias. In addition to dopamine, glutamate excitotoxicity, resulting from disturbance of the homeostatic balance of neurotransmitters and elevated extracellular levels of glutamate, is potentially an important therapeutic target. Safinamide has been investigated in phase III clinical trials as adjunct therapy to I-dopa in mid- to late-stage fluctuating PD. Adding safinamide to I-dopa increases the time patients' symptoms are controlled - so-called ON time, without increasing troublesome dyskinesia. Although safinamide has dopaminergic actions, recent data have suggested that the long-term effects of safinamide on dyskinesia are related to safinamide state- and use-dependent inhibition of sodium channels and stimulated glutamate release, rather than reduced dopaminergic stimulation. Safinamide's unique dual mechanism of action makes it a valuable treatment option for fluctuating PD patients.

\section{Keywords}

Dopamine, glutamate, Parkinson's disease, safinamide

Disclosure: Jaime Kulisevsky has received honoraria for lecturing or advisory boards from UCB, Zambon, Lundbeck and Abbvie, and research support from Instituto de Salud Carlos III, Spain. This article involves a review of the literature and did not involve any studies with human or animal subjects performed by any of the authors.

Acknowledgements: Medical writing assistance was provided by Katrina Mountfort at Touch Medical Media, funded by Zambon SpA

Authorship: All named authors meet the International Committee of Medical Journal Editors (ICMJE) criteria for authorship of this manuscript, take responsibility for the integrity of the work as a whole, and have given final approval to the version to be published.

open Access: This article is published under the Creative Commons Attribution Noncommercial License which permits any non-commercial use, distribution, adaptation and reproduction provided the original author(s) and source are given appropriate credit.

Received: 15 August 2016

Accepted: 4 October 2016

Citation: European Neurological Review,

2016;11(2):101-5

Corresponding Author: Jaime Kulisevsky, Movement Disorders Unit, Neurology Department, Sant Pau Hospital Universitat Autónoma de Barcelona, Mas Casanovas 9008041 Barcelona, Spain. E: jkulisevsky@santpau.cat

Support: The publication of this article was funded by Zambon SpA. The views and opinions expressed are those of the author and do not necessarily reflect those of Zambon SpA.
Parkinson's disease $(\mathrm{PD})$ is a progressive neurodegenerative disorder, with a prevalence that increases with age: 41 in 100,000 in the age group 40-49, rising to 1,903 in 100,000 in those aged over 80 years. ${ }^{1}$ PD is characterised by striatal dopamine deficiency resulting from progressive degeneration of dopaminergic cells in the pars compacta of the substantia nigra, the brain region responsible for motor control.2 Symptoms of PD include bradykinesia, rigidity, tremor, gait and postural abnormalities, and speech difficulty, ${ }^{3}$ as well as non-motor symptoms including cognitive dysfunction, mood disorders, sleep disturbance and pain. ${ }^{4}$

Although PD is incurable, symptoms can be alleviated using dopaminergic therapies including the dopamine precursor levodopa (I-dopa), dopamine agonists and monoamine oxidase (MAO)-B inhibitors that block dopamine degradation, thereby helping restore dopamine levels in the brain. ${ }^{5,6} \mathrm{~L}$-dopa is currently the standard treatment for motor symptoms in PD, but its long-term use has important limitations. ${ }^{5}$ As the disease progresses, the response to I-dopa doses becomes shorter and patients motor fluctuations, which involve periods of being ON, during which the patient experiences a positive response to medication, and being OFF, during which the patient experiences a re-emergence of the symptoms suppressed during the ON state. ${ }^{7.8}$ Furthermore, symptoms of PD, including gait and tremor, gradually become resistant to I-dopa. ${ }^{8}$ Other limitations of the use of I-dopa include the fact that it does not prevent PD progression, ${ }^{\prime}$ and many non-motor symptoms do not respond well to I-dopa.?

As well as motor fluctuations, abnormal involuntary movements, known as I-dopa-induced dyskinesia (LID), develop with prolonged use of I-dopa; these may occur at peak effect of I-dopa, at the beginning and end of dose, or between doses (see Figure 1). ${ }^{8,10-12}$ These movements usually involve the face and the side of the body that is initially affected by the disease, but they commonly progress and affect the neck, upper and lower limbs, and body axis. Chorea and dystonia are the most frequent forms of LIDs, but ballismus and myoclonus can also appear. ${ }^{13,14}$ Around $40 \%$ of patients experience motor fluctuations and LID after 4-6 years of treatment with I-dopa, ${ }^{14}$ and 60\% to $100 \%$ may experience LID after 10 years..$^{15,16}$

Motor fluctuations are the result of alterations of the functional organisation of the basal ganglia circuitry following long-term exposure to I-dopa. Progressive degeneration of the nigro-striatal dopaminergic pathway reduces the ability of nerve terminals to store and release dopamine. ${ }^{17}$ As a result of this loss of storage capacity, I-dopa induces a more pulsatile stimulation of postsynaptic dopamine receptors, consistent with I-dopa's short (90-minute) half-life and its rapid cycling pharmacokinetics. This pulsatile stimulation causes functional changes within the basal ganglia, leading to involuntary movements and altered neural activity in the basal ganglia, thalamus cerebral cortex.17 
Figure 1: Estimated frequency of different types of levodopa-induced dyskinesia

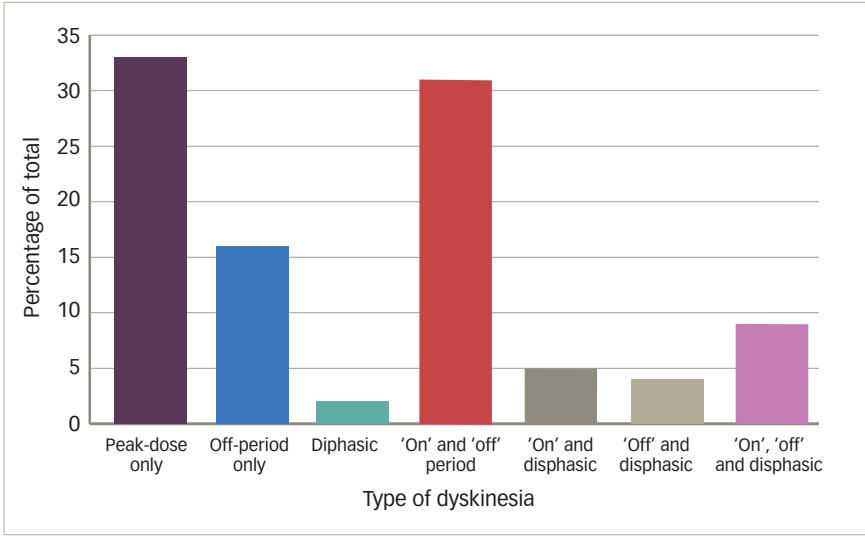

Source: Aquino, $2015^{8}$

Figure 2: Dual mechanism of action of safinamide

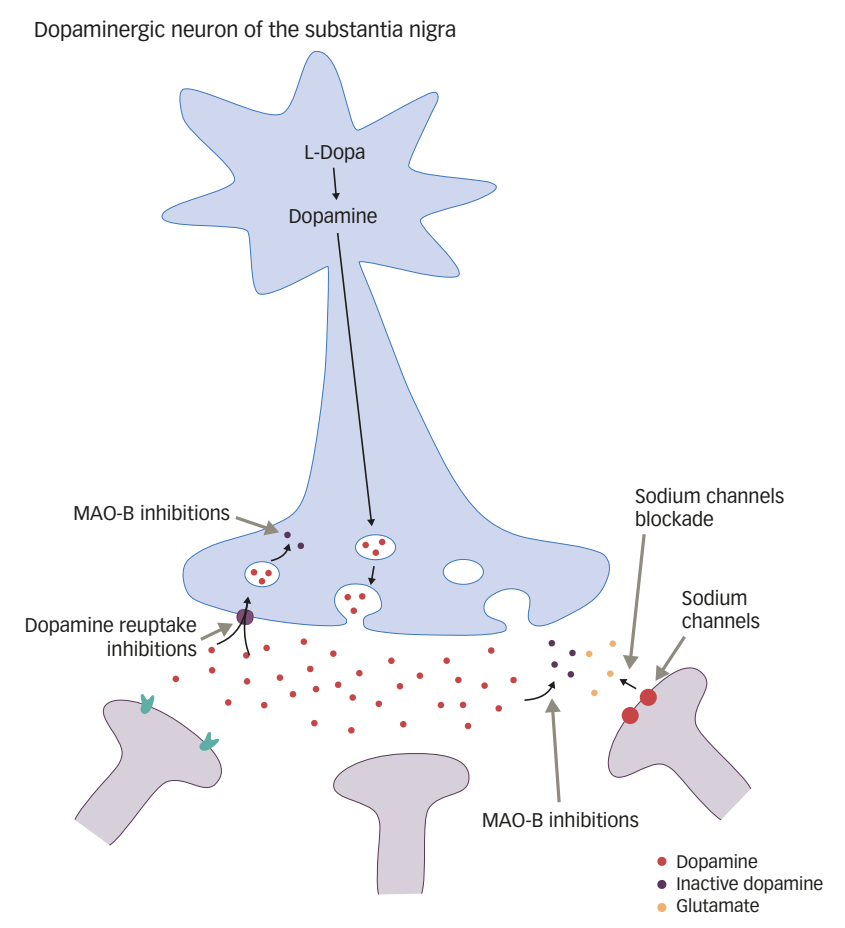

L-dopa = levodopa; $M A O-B=$ monoamine oxidase $-B$

Several risk factors are associated with the development of LID, including increased body weight, ${ }^{18}$ age of onset and severity of PD, ${ }^{19}$ higher dose and longer duration of I-dopa therapy.,19 Dyskinesias are associated with substantially decreased quality of life (QoL) for both patients and caregivers, and create an additional burden on healthcare systems as they require increased monitoring and complex medical management. ${ }^{8,20}$ Moreover, as LIDs worsen, they lead to increased risk of injury, exhaustion and fatigue. ${ }^{8}$

Patients with motor fluctuations often require add-on therapies, many of which improve motor fluctuations, but exacerbate dyskinesia. ${ }^{21}$ The current recommended treatment for dyskinesia is the non-selective $\mathrm{N}$-methyl-D-aspartate (NMDA) receptor antagonist amantadine, ${ }^{22}$ but its effects are modest and short in duration. Moreover, it is associated with poor tolerance and adverse effects including hallucinations, leg oedema and livedo reticularis, requiring careful monitoring. ${ }^{2,23}$
Clozapine, a high-affinity serotoninergic agonist used to treat psychosis in PD, has also been found to reduce LID $^{24}$ but it is associated with severe adverse effects, ${ }^{25,26}$ that limit its use in this indication. Non-oral treatment includes continuous subcutaneous apomorphine infusion, ${ }^{27}$ levodopa-carbidopa intestinal ge ${ }^{28}$ and transdermal rotigotine therapy. ${ }^{29}$ Deep-brain stimulation may help improve motor fluctuations and dyskinesia in advanced PD. ${ }^{30}$

In a 2009 survey, better treatments for motor symptoms were emphasised as a major unmet need in PD therapy. ${ }^{31}$ Fluctuating response to treatment is the most troublesome motor symptom reported by PD patients. ${ }^{32}$ There is therefore an unmet need for additional therapeutic options in fluctuating PD patients.

\section{Pathophysiological mechanisms underlying dyskinesias in Parkinson's disease}

While the pathogenesis of motor fluctuations has not been fully elucidated, in vitro studies suggest that other neurotransmitters in the brain, including glutamate, adenosine and serotonin are involved in the control of motor symptoms and the development of dyskinesia. ${ }^{11}$ Glutamate plays a key role in the pathophysiology of PD. The dendritic spines of striatal medium-sized gamma-aminobutyric acid (GABA)ergic neurons exhibit both dopamine and $\mathrm{N}$-methyl-D-aspartate (NMDA) receptors and receive both cortical-striatal glutamatergic projections and nigrostriatal dopaminergic fibres. ${ }^{33}$ Excessive oxidative stress in the substantia nigra pars compacta results in a reduction in the capacity of nigral neurons to cope with metabolic demands and the development of susceptibility to the effects of glutamate. Under conditions of impaired cellular energy metabolism, glutamate acts as a neurotoxin, causing degeneration of nigral neurons followed by striatal dopaminergic denervation. ${ }^{33}$ This leads to a pathophysiological cascade of 1-methyl-4-phenyl-1,2,3,6-tetrahydropyridine (MTMP)-induced neuronal cell death. ${ }^{34}$ The loss of dopamine neurons is believed to cause an increase in glutamatergic activity in the basal ganglia. ${ }^{35}$ This activates the striatopallidal pathway, inhibiting outputs of the basal ganglia and resulting in dyskinesia. ${ }^{36}$ Glutamate excitotoxicity is therefore potentially an important therapeutic target. ${ }^{37}$ Targeting non-dopaminergic systems provides complementary approach that may improve and control motor complications, while maintaining the efficacy of I-dopa. ${ }^{38}$

\section{The use of safinamide in Parkinson's disease}

Safinamide (Xadago ${ }^{\circledR}$, Zambon, Bresso, Italy) is a novel $\alpha$-aminoamide with a unique multi-modal mechanism of action distinct from that of amantadine. ${ }^{39}$ It has antiparkinsonian, anticonvulsant and neuroprotective properties. Safinamide offers a unique approach to the management of motor symptoms and motor fluctuation/dyskinesia due to its unique dual mechanism of action (see Figure 2). Safinamide has dopaminergic mechanisms of action, including selective and reversible MAO-B and dopamine reuptake inhibition, which are largely responsible for its effects on motor symptoms. ${ }^{39-41}$ However, it also has nondopaminergic mechanisms of action. It suppresses repetitive firing by useand state-dependent sodium channel blockade that in turn decreases calcium channel opening, and reduces excessive release from synaptic terminals of glutamate. ${ }^{42}$ In this glutamatergic mechanism of action, safinamide differs from amantadine and memantine; it has an indirect effect on glutamate release through sodium channels blockade, whereas amantadine and memantine have a direct effect due to NMDA receptor antagonism. ${ }^{39,43,44}$ It must be stressed that safinamide has a multimodal action and is not a pure antidyskinetic drug. In addition, safinamide has no effect on I-type calcium channels, and therefore does not affect blood pressure or heart rate..$^{45}$ 
Table 1: Summary of clinical trials to date investigating the use of safinamide in Parkinson's disease as add-on to levodopa

\begin{tabular}{|c|c|c|c|c|}
\hline Study name & Patient population & Study design & Efficacy findings & Safety findings \\
\hline Study $016^{49}$ & $\begin{array}{l}\text { 6-month (24-week) trial, } \\
n=669, \text { PD fluctuating } \\
\text { patients, receiving a } \\
\text { stable dose of levodopa } \\
\text { (alone or with other } \\
\text { anti-parkinson drugs) }\end{array}$ & $\begin{array}{l}\text { Patients received placebo, } \\
\text { safinamide } 50 \text { or } 100 \text { mg/ } \\
\text { day as add-on to levodopa }\end{array}$ & $\begin{array}{l}\text { At week } 24 \text {, improvements in ON time with no/ } \\
\text { non-troublesome dyskinesia and OFF time were } \\
\text { significantly higher in safinamide } 50 \text { and } 100 \text { mg/ } \\
\text { day groups versus. placebo, starting from week } \\
4 \text { onward, as well as improvements in UPDRS III } \\
\text { motor scores }\end{array}$ & $\begin{array}{l}\text { There were no significant differences among } \\
\text { groups in the incidence of SAES, TEAEs or } \\
\text { AEs causing discontinuation. Most common } \\
\text { AEs ( } \geq 5 \% \text { in one group or more) were } \\
\text { non-troublesome dyskinesia, back pain, } \\
\text { worsening of PD, cataract and headache }\end{array}$ \\
\hline Study $018^{50}$ & $\begin{array}{l}\text { 18-month extension } \\
\text { of study } 016, n=544\end{array}$ & $\begin{array}{l}\text { Patients continued on } \\
\text { randomised placebo, } \\
50 \text {, or } 100 \mathrm{mg} / \text { day } \\
\text { safinamide }\end{array}$ & $\begin{array}{l}\text { Change in DRS was not significantly different in } \\
\text { safinamide vs. placebo groups. Ad hoc subgroup } \\
\text { analysis of moderate to severe dyskinetic patients } \\
\text { at baseline ( } 36 \% \text { of patients) showed a statistically } \\
\text { significant decrease with safinamide } 100 \text { mg/day } \\
\text { vs. placebo. Improvements in ON and OFF time, } \\
\text { motor function, activities of daily living and QoL } \\
\text { at } 6 \text { months remained significant at } 24 \text { months }\end{array}$ & $\begin{array}{l}\text { SAES, TEAES and discontinuation rates were } \\
\text { similar with safinamide and placebo }\end{array}$ \\
\hline SETTLE $^{52}$ & $\begin{array}{l}\text { 6-month (24-week) trial, } \\
\mathrm{n}=552, \mathrm{PD} \text { fluctuating } \\
\text { patients, receiving a } \\
\text { stable dose of levodopa } \\
\text { (alone or with other } \\
\text { anti-Parkinson drugs) }\end{array}$ & $\begin{array}{l}\text { Patients started treatment } \\
\text { with safinamide } 50 \mathrm{mg} / \\
\text { day and could increase } \\
\text { the dose to } 100 \mathrm{mg} / \text { day } \\
\text { after } 2 \text { weeks. Patients } \\
\text { received placebo or } \\
\text { safinamide as add-on to } \\
\text { levodopa }\end{array}$ & $\begin{array}{l}\text { At } 24 \text { weeks, significantly more patients in the } \\
\text { safinamide group vs placebo group had an } \\
\text { improvement in both ON time with no/non- } \\
\text { troublesome dyskinesia, OFF time and in the } \\
\text { UPDRS III motor score }\end{array}$ & $\begin{array}{l}\text { TEAES, discontinuation rate and SAES were } \\
\text { similar across treatments. The most frequent } \\
\text { treatment-related AEs ( } \geq 5 \% \text { in one group } \\
\text { or more) were back pain, non-troublesome } \\
\text { dyskinesia, falls, headache, nausea, and } \\
\text { urinary tract infections }\end{array}$ \\
\hline
\end{tabular}

$A E=$ adverse effect; $D R S=$ Dyskinesia Rating Scale; $P D=$ Parkinson's disease; $Q$ L = quality of life; SAE = serious adverse effect; TEAE = treatment-emergent adverse effect; UPDRS = unified Parkinson's disease rating scale.

Studies investigating safinamide's anticonvulsant properties in rat brain membranes showed that safinamide has high affinity for binding site 2 of the sodium channel receptor and exerts its actions through the inhibition of sodium and calcium channels, stabilising neuronal membrane excitability and inhibiting transmitter release. ${ }^{46}$ A higher number of sodium channels are therefore kept in the inactivated state. In another animal study, the sodium channel opener, veratridine, was administered for 30 minutes to induce a rapid and transient increased glutamate release in the rat hippocampus. Safinamide inhibited glutamate release without affecting basal glutamate release. ${ }^{47}$

This non-dopaminergic mechanism of action of safinamide might be important in terms of the effects of the drug on motor complications. In a study of a primate model, both safinamide and amantadine reduced LID but only safinamide increased the duration of response of I-dopa. ${ }^{48}$ These effects cannot be accounted only for by MAO-B inhibition, since the latter is associated with worsening rather than improvement of LID. The authors concluded that this finding might be related to a reduction in cortical and/or thalamic excitatory inputs resulting from reduced presynaptic glutamate release due to the blockade of voltage-gated sodium channels.

Safinamide has emerged as a useful add-on therapy for I-dopa following the findings of three phase III multicentre, randomised, double-blind, placebo-controlled, parallel-group trials of patients with mid-to latestage PD and motor fluctuations: trials 016, 018 and SETTLE (Safinamide in Idiopathic Parkinson's Disease With Motor Fluctuations, as add-on to Levodopa). ${ }^{49-51}$ In all studies, treatment with safinamide at daily doses of 50-100 mg (studies 016 and 018) or $100 \mathrm{mg}$ (SETTLE) for 24 weeks (studies 016 and SETTLE) or 2 years (study 018) as add-on to I-dopa (alone or with other anti-parkinson drugs) increased daily ON time without dyskinesia, as assessed by Hauser patient diaries (see Table 1). ${ }^{51}$ The dose of I-dopa remained stable in the majority of patients.
In study 016 ( $n=669)$, there were significant differences in the mean change in ON time without dyskinesia versus placebo in both the safinamide $50 \mathrm{mg}$ (0.51 hours; $95 \%$ confidence interval [Cl] 0.07-0.94; $\mathrm{p}=0.0223)$ and the safinamide $100 \mathrm{mg}(0.55$ hours; $95 \% \mathrm{Cl} 0.12-0.99$; $\mathrm{p}=0.0130$ ) groups. Both safinamide doses did not increase troublesome dyskinesia despite the significant increase in ON time. There were also significant improvements in OFF time and Unified Parkinson's Disease Rating Scale (UPDRS)-III (motor) scores. In the $100 \mathrm{mg}$ dosage group there were significant increases in UPDRS-II (activities of daily living) scores, Parkinson's Disease Questionnaire (PDQ-39) total score $(p=0.0360)$ and subscale scores for emotional wellbeing ( $p=0.0116)$, communication $(\mathrm{p}=0.0361)$, and bodily discomfort. Differences from placebo in $\mathrm{ON}$ and OFF time were significant for both doses $(p=0.0159)$ from the first postbaseline evaluation (week 4) onward. ${ }^{49}$

Of the patients enrolled in study $016,81 \%(n=544)$ continued into an 18-month extension study (study 018). ${ }^{50}$ Inclusion criteria for continuation were compliance with treatment and willingness to continue, or discontinuation but completion of the scheduled efficacy evaluations at weeks 12 and 24. Patients were excluded if they had experienced clinically significant adverse events (AEs) or shown clinically significant deterioration in motor symptoms during study 016. The primary endpoint was the change from baseline in Dyskinesia Rating Scale (DRS) total score during ON time over 24 months. The improvements in ON time without dyskinesia seen at six months in study 016 were maintained up to two years in study 018 , as well as the improvements in OFF time and in motor scores. Although there was no overall difference in dyskinesias between patients and controls, improvement in dyskinesia compared with placebo $(p=0.0317)$ was seen in patients at least moderately dyskinetic (36\% of patients) at baseline. ${ }^{50}$

In the SETTLE study ( $n=552)$, as early as week 2 (first post-baseline assessment), a significant increase in median ON time without dyskinesia 
Figure 3: Proportions of patients with different categorical changes in dyskinesia rating scale score (decrease, no change, increase) and no change in their levodopa dose (post-hoc analysis)

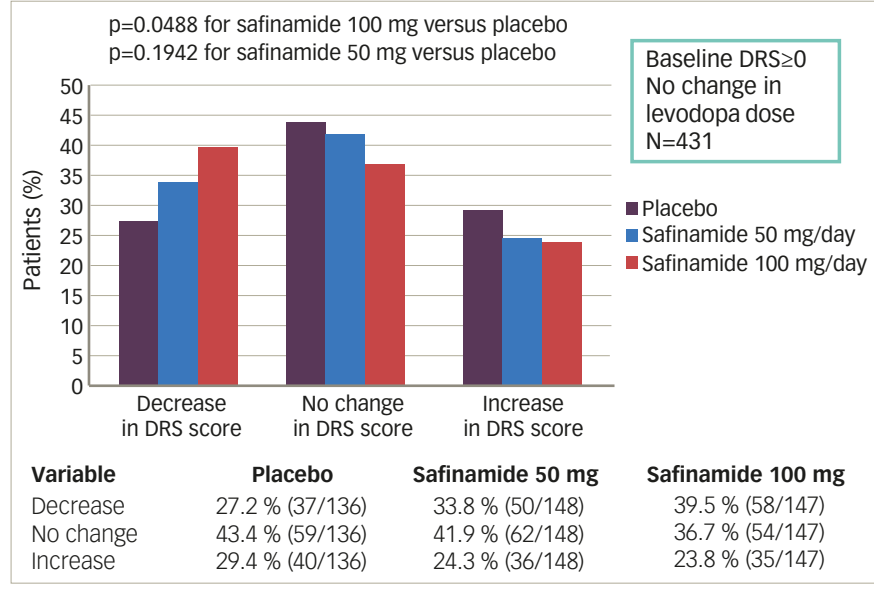

DRS = Dyskinesia Rating Scale. Reproduced with permission from Cattaneo, $2015^{54}$

and OFF time of one hour was observed for safinamide (50 mg/day) compared with 30 minutes for placebo..11 At week 24, the change from baseline to 24 weeks in daily ON time with no/non-troublesome dyskinesia was significantly different for safinamide $100 \mathrm{mg} /$ day versus placebo (0.96 hours; $95 \% \mathrm{Cl} 0.56-1.37 ; \mathrm{p}<0.001)$. Safinamide also significantly improved UPDRS-III score, Clinical Global Impression of Severity (CGI-S), Clinical Global Impression of Change (CGI-C), the 39-item PDQ-39, and OFF time following the first morning I-dopa dose (i.e., latency to $\mathrm{ON}$ ) compared to placebo. In addition, a significantly higher proportion of patients achieved improvements of at least one hour in motor fluctuations and a percentage of improvement of 30\% and more in motor symptoms, a clinically meaningful benefit. ${ }^{52}$

Safinamide was well tolerated in these studies. Common adverse effects (reported in $\geq 5 \%$ of patients) included worsening of PD, cataract, back pain, pyrexia and hypertension. ${ }^{49,52}$ In study 016 , dose reduction was required for 10 patients (4\%) in the $100 \mathrm{mg} /$ day group, seven (3\%) in the $50 \mathrm{mg}$ /day group, and nine (4\%) in the placebo group. Completion rates were high (87\%-90\%), and there were no significant betweengroup differences for incidences of treatment-emergent AES (TEAES) or TEAEs leading to discontinuation. Although dyskinesia was reported as a TEAE in a minority of patients, this was generally mild or moderate. ${ }^{49}$ This seems an incongruous finding, since patient-recorded ON time showed that safinamide significantly improved ON time with no or non-troublesome dyskinesia, and may be due to differences in data collection. Patients recorded ON time in their diaries every 30 minutes over an 18-hour period for five consecutive days preceding each visit. By contrast, AEs were reported at study visits and may reflect whether a patient is experiencing any dyskinesia at that moment, regardless of its impact on functioning.

As a result of these data, safinamide received European Medicines Agency approval in 2015, (dosage 50-100 mg/day) for the treatment of mid- to late-stage fluctuating PD, as an add-on to I-dopa, alone or in combination with other PD medications..$^{53}$

A post-hoc analysis of studies 016 and 018 aimed to characterise further the effects of safinamide on dyskinesia in mid- to late-stage PD patients. ${ }^{54}$ While the effects of the $50 \mathrm{mg} /$ day dosage of safinamide in study 016 may be attributable to MAO-B inhibition, the superiority of the effects at $100 \mathrm{mg} /$ day suggest that other mechanisms may be involved, as a dose of $50 \mathrm{mg} /$ day is sufficient to achieve complete MAO-B inhibition. It was therefore postulated that the antidyskinetic effects of the higher dose could be attributed to non-dopaminergic mechanisms. Patients were therefore stratified by the presence or absence of dyskinesia at baseline score $>0$ or DRS score $=0$, respectively), and by whether or not the dose of I-dopa had been altered during the 24-month treatment period. Safinamide $100 \mathrm{mg} /$ day significantly improved the DRS versus placebo in the subgroup of patients with or without changes in I-dopa dose. In patients with baseline dyskinesia, improvements were also significant in patients without changes in I-dopa dose (see Figure 3), suggesting that these improvements did not result from reduced dosage of I-dopa, but from the action of safinamide $100 \mathrm{mg}$ on glutamatergic neurotransmission. The authors concluded that the higher dose is needed to optimise the dopaminergic and non-dopaminergic effects of safinamide. ${ }^{54}$

A post-hoc analysis of pooled data from studies 016 and SETTLE found that safinamide $100 \mathrm{mg}$ significantly increased mean ON time with no or non-troublesome dyskinesia by $1.42 \mathrm{~h}$ ( $95 \% \mathrm{Cl}: 1.21$ to 1.64 ) versus 0.58 hour $(0.37$ to 0.80$)$ with placebo $(p<0.0001)$ and reduced mean OFF time by -1.49 hour $(-1.68$ to -1.30 ) versus -0.63 hour $(-0.82$ to -0.44$)$ with placebo; $p<0.0001)$. Significant changes compared with placebo in $\mathrm{ON}$ and OFF time were observed whether safinamide used as first or later adjunct therapy in I-dopa treated patients, and regardless of whether patients were receiving concomitant dopamine agonists, catechol-O-methyltransferase inhibitors or amantadine. ${ }^{55}$ These findings suggest that safinamide may be considered either as a first adjunct therapy in PD patients who are not sufficiently controlled on I-dopa, and as an add-on therapy in patients taking I-dopa and other concomitant dopaminergic medications.

There is a need for further studies to explore the effect of safinamide on LID, as it is an important finding. Patients consider ON time with no or non-troublesome dyskinesia as 'good' ON time and it correlates with patients' perceived duration of a good response throughout the day. ${ }^{49}$ By contrast, the MAO inhibitor rasagiline has shown to increase ON time with troublesome dyskinesia, suggesting that targeting the dopaminergic pathway alone is effective in controlling motor fluctuations but not motor complications. ${ }^{42}$ In the phase III PRESTO study, the difference between the rasagiline dose of $1.0 \mathrm{mg} /$ day and placebo in the ON time with dyskinesia was in favour of placebo: $0.37 \mathrm{~h}(\mathrm{p}=0.048) .{ }^{56}$

\section{Summary and concluding remarks}

PD is a complex condition and recent advances have elucidated the pathogenic processes underlying its symptoms. Numerous nondopaminergic neurotransmitters are involved in the control of motor symptoms and the development of motor fluctuations and dyskinesias following long-term l-dopa therapy. In particular, glutamate plays a key role in the pathophysiology of PD and represents a valuable therapeutic target.

Unlike other drugs that can improve motor function, long-term studies have shown that safinamide does not lead to an overall worsening of dyskinesia. Recent data suggest that this may be a consequence of its a unique dual mechanism of action, combining reversible MAO-B inhibition with blockage of voltage-dependent sodium channels, modulation of calcium channels and inhibition of abnormal glutamate release. The inhibition of sodium and calcium channels by safinamide is an important clinical finding and warrants further investigation. In phase III clinical studies, safinamide has been shown to significantly increase ON time with no/non-troublesome dyskinesias and decrease OFF time, as well as having beneficial effects on motor symptoms and QoL. Safinamide has 
been investigated in long-term (24-month) studies, and has shown a very good safety profile, as well as long-term efficacy.

In conclusion, safinamide is a unique therapy that combines nondopaminergic and dopaminergic mechanisms of action, modulating altered dopaminergic and glutamatergic neurotransmission. There is a need for more clinical trial data to assess the effect of safinamide in dyskinesias. However, clinical trial findings to date suggest that safinamide represents an important therapeutic option for fluctuating PD patients. $\square$
1. 1. Pringsheim T, Jette N, Frolkis A, et al., The prevalence of Parkinson's disease: a systematic review and meta-analysis, Mov Disord, 2014:29:1583-90

2. EMC, Amantadine hydrochloride: SPC. Available at: www. medicines.org.uk/emc/medicine/32086 (accessed 19 July 2016).

3. Lang AE, Lozano AM, Parkinson's disease. First of two parts, $N$ Eng/ J Med, 1998:339:1044-53.

4. Chaudhuri KR, Schapira AH, Non-motor symptoms of Parkinson's disease: dopaminergic pathophysiology and treatment, Lancet Neurol, 2009:8:464-74.

5. Grosset DG, Macphee GJ, Nairn M, et al., Diagnosis and pharmacological management of Parkinson's disease: summary of SIGN guidelines, BMJ, 2010;340:b5614.

6. Birkmayer W, Hornykiewicz O, The effect of I-3,4dihydroxyphenylalanine (=DOPA) on akinesia in parkinsonism, Parkinsonism Relat Disord, 1998;4:59-60.

7. Jenner P, Wearing off, dyskinesia, and the use of continuous drug delivery in Parkinson's disease, Neurol Clin, 2013;31:S17-35.

8. Aquino CC, Fox SH, Clinical spectrum of levodopa-induced complications, Mov Disord, 2015;30:80-9.

9. Olanow CW, Agid Y, Mizuno Y, et al., Levodopa in the treatment of Parkinson's disease: current controversies, Mov Disord, 2004:19:997-1005.

10. Chase TN, Holden EM, Brody JA, Levodopa-induced dyskinesias Comparison in Parkinsonism-dementia and amyotrophic lateral sclerosis, Arch Neurol, 1973;29:328-33.

11. Huot P, Johnston TH, Koprich JB, et al., The pharmacology of L-DOPA-induced dyskinesia in Parkinson's disease, Pharmacol ReV, 2013;65:171-222

12. Thanvi B, Lo N, Robinson T, Levodopa-induced dyskinesia in Parkinson's disease: clinical features, pathogenesis, prevention and treatment, Postgrad Med J, 2007;83:384-8.

13. Bargiotas P, Konitsiotis $\mathrm{S}$, Levodopa-induced dyskinesias in Parkinson's disease: emerging treatments, Neuropsychiatr Dis Treat, 2013;9:1605-17.

14. Ahlskog JE, Muenter MD, Frequency of levodopa-related dyskinesias and motor fluctuations as estimated from the cumulative literature, Mov Disord, 2001;16:448-58.

15. Rascol O, Brooks DJ, Korczyn AD, et al., A five-year study of the incidence of dyskinesia in patients with early Parkinson's disease who were treated with ropinirole or levodopa, $N$ Eng/ $\rfloor$ Med, 2000:342:1484-91.

16. Van Gerpen JA, Kumar N, Bower JH, et al., Levodopa-associated dyskinesia risk among Parkinson disease patients in Olmsted County, Minnesota, 1976-1990, Arch Neurol, 2006;63:205-9.

17. de la Fuente-Fernandez R, Sossi V, Huang Z, et al., Levodopainduced changes in synaptic dopamine levels increase with progression of Parkinson's disease: implications for dyskinesias, Brain, 2004;127:2747-54

18. Sharma JC, Macnamara L, Hasoon M, et al., Cascade of levodopa dose and weight-related dyskinesia in Parkinson's disease (ID-WD-PD cascade), Parkinsonism Relat Disord, 2006;12:499-505.

19. Schrag A, Quinn N, Dyskinesias and motor fluctuations in Parkinson's disease. A community-based study, Brain, 2000;123 (Pt 11):2297-305.

20. Chapuis S, Ouchchane L, Metz O, et al., Impact of the motor complications of Parkinson's disease on the quality of life, MOV Disord, 2005;20:224-30.

21. Horstink M, Tolosa E, Bonuccelli U, et al., Review of the therapeutic management of Parkinson's disease. Report of a joint task force of the European Federation of Neurological Societies (EFNS) and the Movement Disorder Society-European Section (MDS-ES). Part II: late (complicated) Parkinson's disease Eur J Neurol, 2006;13:1186-202.

22. Fox SH, Katzenschlager, R. Lim, S.Y., et al.., The Movement Disorder Society Evidence-Based Medicine Review Update: Treatments for the motor symptoms of Parkinson's disease Mov Disord, 2011:26:Suppl 3:S2-4.

23. Antonini A, Tolosa $E$, Apomorphine and levodopa infusion therapies for advanced Parkinson's disease: selection criteria and patient management, Expert Rev Neurother 2009;9:859-67.

24. Durif F, Debilly B, Galitzky M, et al., Clozapine improves dyskinesias in Parkinson disease: a double-blind, placebocontrolled study, Neurology, 2004;62:381-8.

25. Haas SJ, Hill R, Krum H, et al., Clozapine-associated myocarditis: a review of 116 cases of suspected myocarditis associated with the use of clozapine in Australia during 1993-2003, Drug Saf. 2007;30:47-57.

26. Alvir JM, Lieberman JA, Safferman AZ, et al., Clozapine-induced agranulocytosis. Incidence and risk factors in the United States, NEngl I Med, 1993:329:162-7.

27. Katzenschlager R, Hughes A, Evans A, et al., Continuous subcutaneous apomorphine therapy improves dyskinesias in Parkinson's disease: a prospective study using single-dose challenges, Mov Disord, 2005;20:151-7.

28. Olanow CW, Kieburtz K, Odin P, et al., Continuous intrajejunal infusion of levodopa-carbidopa intestinal gel for patients with advanced Parkinson's disease: a randomised, controlled, double-blind, double-dummy study, Lancet Neurol, 2014;13:141-9.

29. LeWitt PA, Lyons KE, Pahwa R, et al., Advanced Parkinson disease treated with rotigotine transdermal system: PREFER Study, Neurology, 2007;68:1262-7.

30. Benabid AL, Deep brain stimulation for Parkinson's disease, Curr Opin Neurobiol, 2003;13:696-706.

31. Hatano T, Kubo SI, Shimo Y, et al., Unmet needs of patients with Parkinson's disease: interview survey of patients and caregivers, I Int Med Res, 2009:37:717-26.

32. Politis M, Wu K, Molloy S, et al., Parkinson's disease symptoms: the patient's perspective, Mov Disord, 2010;25:1646-51.

33. Blandini F, Porter RH, Greenamyre JT, Glutamate and Parkinson's disease, Mol Neurobiol, 1996;12:73-94.

34. Lange KW, Kornhuber J, Riederer P, Dopamine/glutamate interactions in Parkinson's disease, Neurosci Biobehav Rev 1997;21:393-400.

35. Barone P, Neurotransmission in Parkinson's disease: beyond dopamine, Eur J Neurol, 2010;17:364-76.

36. Brotchie JM, Nondopaminergic mechanisms in levodopainduced dyskinesia, Mov Disord, 2005;20:919-31.

37. Jia M, Njapo SA, Rastogi V, et al., Taming glutamate excitotoxicity: strategic pathway modulation for excitotoxicity: strategic pathway modulation
neuroprotection, CNS Drugs, 2015;29:153-62

38. Fox SH, Non-dopaminergic treatments for motor control in Parkinson's disease, Drugs, 2013;73:1405-15.

39. Onofrj M, Bonanni L, Thomas A, An expert opinion on safinamide in Parkinson's disease, Expert Opin Investig Drugs, 2008;17:1115-25

40. Marzo A, Dal Bo L, Monti NC, et al., Pharmacokinetics and pharmacodynamics of safinamide, a neuroprotectant with antiparkinsonian and anticonvulsant activity, Pharmacol Res, 2004;50:77-85

41. Dezsi L, Vecsei L, Safinamide for the treatment of Parkinson's disease, Expert Opin Investig Drugs, 2014;23:729-42.

42. Pevarello $P$, Traquandi $G$, Bonsignori $A$, et al., Synthesis and preliminary biological evaluation of new alpha-amino amide anticonvulsants incorporating a dextromethorphan moiety, Bioorg Med Chem Lett, 1999:9:1783-8.

43. Stoof JC, Booij J, Drukarch B, et al., The anti-parkinsonian drug amantadine inhibits the $\mathrm{N}$-methyl-D-aspartic acid-evoked release of acetylcholine from rat neostriatum in a noncompetitive way, Eur I Pharmacol, 1992;213:439-43.

44. Johnson JW, Kotermanski SE, Mechanism of action of memantine, Curr Opin Pharmacol, 2006;6:61-7.

45. Caccia C, Maj R, Calabresi M, et al., Safinamide: from molecular targets to a new anti-Parkinson drug, Neurology, 2006;67:S18-23

46. Salvati P, Maj R, Caccia C, et al., Biochemical and electrophysiological studies on the mechanism of action of PNU-151774E, a novel antiepileptic compound, J Pharmacol Exp Ther, 1999:288:1151-9.

47. Melloni E, Brugnoli, E., Caccia, C. et al Safinamide and glutamate release: new insights, Parkinsonism Relat Disord, 2016;22:e177.

48. Gregoire L, Jourdain VA, Townsend M, et al., Safinamide reduces dyskinesias and prolongs L-DOPA antiparkinsonian effect in parkinsonian monkeys, Parkinsonism Relat Disord, 2013;19:508-14

49. Borgohain R, Szasz J, Stanzione P, et al., Randomized trial of safinamide add-on to levodopa in Parkinson's disease with motor fluctuations, Mov Disord, 2014;29:229-37.

50. Borgohain R, Szasz J, Stanzione P, et al., Two-year, randomized, controlled study of safinamide as add-on to levodopa in mid to late Parkinson's disease, Mov Disord, 2014;29:1273-80.

51. Anand R, Lucini V, Forrest E, et al., Early onset of efficacy of safinamide on motor fluctuations in PD patients on L-dopa and other PD medications (SETTLE study), Mov Disord, 2014;29:1273-80.

52. Schapira A, Fox S, Hauser RA, et al., Safinamide add on to L-dopa: a randomized, placebo-controlled, 24-week globa trial in patients with Parkinson's Disease (PD) and motor fluctuations (SETTLE), Presented at the 65th American Academy of Neurology Annual Meeting, San Diego, CA, USA, 16-23 March 2013.

53. EMA, Safinamide: Summary of Product Characteristics. Available at: www.ema.europa.eu/docs/en_GB/document library/EPAR__Product_Information/human/002396/ WC500184965.pdf (accessed 1 July 2016).

54. Cattaneo C, Ferla RL, Bonizzoni E, et al., Long-Term Effects of Safinamide on Dyskinesia in Mid- to Late-Stage Parkinson's Disease: A Post-Hoc Analysis, J Parkinsons Dis, 2015;5:475-81.

55. Cattaneo C, Sardina M, Bonizzoni E, Safinamide as Add-On Therapy to Levodopa in Mid- to Late-Stage Parkinson's Disease Fluctuating Patients: Post hoc Analyses of Studies 016 and Fluctuating Patients: Post hoc Analyses of

56. Parkinson Study Group, A randomized placebo-controlled trial of rasagiline in levodopa-treated patients with Parkinson disease and motor fluctuations: the PRESTO study, Arch Neurol, 2005;62:241-8. 